\title{
Эффект твердофазной адсорбции в диффрузионной кинетике селективного растворения сплавов
}

\author{
Козадеров О.А., Введенский А.В., Селеменев В.Ф. \\ ФГБОУ ВО «Воронежский государственный университет», Воронеж
}

Поступила в редакцию 26.10.2016 г.

\begin{abstract}
Применительно к потенциостатическому режиму поляризации при анодном селективном растворении гомогенного сплава поставлена и аналитически решена задача нестационарной диффузии, учитывающая равновесную твердофазную адсорбцию компонентов. Получены аналитические выражения для концентрационного поля и токового транзиента электроотрицательного компонента сплава. Установлено, что учет эффекта твердофазной адсорбции в случае хроноамперометрии избирательно растворяющихся Ag,Au-сплавов, построенных на основе серебра, приводит к увеличению расчетных значений коэффициента диффузии атомов $\mathrm{Ag}$ в сплаве. Влияние предполяризационного твердофазного адсорбционного накопления золота в поверхностном слое Ag,Au-сплава тем менее заметно, чем выше содержание серебра в объеме твердого раствора.
\end{abstract}

Ключевые слова: сплав, селективное растворение, твердофазная адсорбция, нестационарная диффузия, математическое моделирование.

\section{Solid-phase adsorption effect in the diffusion kinetics of selective dissolution of alloys}

\author{
Kozaderov O.A., Vvedenskii A.V., Selemenev V.F. \\ Voronezh State University, Voronezh
}

\begin{abstract}
The purpose of the paper is to solve the problem of non-stationary diffusion at potentiostatic selective dissolution of a homogeneous alloy, accompanied by preliminary solid-phase adsorptive accumulation of a component in the surface layer. The analytical expressions for the concentration field and the transient current of an electronegative alloy component are obtained analytically by means of integral Laplace-Carson transformation method. It was shown that the function of equilibrium adsorption isotherm is completely responsible for the contribution of the pre-polarization segregating the components into the value of the partial current of selective dissolution controlled by solid-state diffusion in the alloy. Mathematical modeling of the solid-phase adsorption effect during anodic selective dissolution of a homogeneous alloy in the mode of diffusion kinetics shows that the change in the surface concentration results in a proportional change in the slope of the criterion Cottrell transient current. The results of the theoretical analysis were used in the treatment of experimental data obtained for Ag-Au alloys. Their advantage is that the isotherm of equilibrium solid-phase adsorption of components of such metallic systems on their boundary with an aqueous solution of surface-inactive anions is known. It has been established experimentally that taking the effect of solid-phase adsorption into account in the case of chronoamperometry of selectively dissolving Ag-Au alloys based on silver in nitrate electrolyte results in an increase in the calculated values of the solid-state diffusion coefficients. The important result is that the pre-polarization solid-adsorptive accumulation of gold in the surface layer of these alloys is the less noticeable the higher the content of silver in the bulk alloy.

Keywords: alloy, selective dissolution, solid-phase adsorption, non-stationary diffusion, mathematical simulation
\end{abstract}




\section{Введение}

В ходе селективного растворения (СР) бинарных сплавов только электроотрицательный компонент А подвергается окислению с переходом простых ионов в раствор, тогда как более благородный металл В остается электрохимически стабильным [1]. В наиболее общем виде процесс может быть описан следующим уравнением:

$$
\mathrm{A}-\mathrm{B} \stackrel{-\mathrm{n}_{\mathrm{A}} \mathrm{e}^{-}}{\longrightarrow} \mathrm{A}^{\mathrm{n}_{\mathrm{A}}^{+}}+\mathrm{B} .
$$

Фактически он представляет собой необратимый распад твердого раствора, реализуемый в условиях анодной поляризации либо коррозионного саморастворения сплава в агрессивной окислительной среде.

При этом скорости перехода компонентов сплава в электролит и их объемные концентрации в фазе твердого раствора не пропорциональны друг другу, что означает относительное обогащение поверхностного слоя сплава более стабильным компонентом. В случае истинно селективного растворения поверхность сплава будет обедняться электроотрицательным металлом А, в то время как поверхностная концентрация металла В в сплаве будет возрастать. Однако даже в отсутствие СР при термодинамическом равновесии поверхностная $\left(x_{\mathrm{i}}^{\mathrm{s}}\right)$ и объемная $\left(x_{\mathrm{i}}^{\mathrm{v}}\right)$ атомные доли i-го компонента сплава могут быть различными [2]. Причиной такой поверхностной сегрегации является особое энергетическое состояние атомов в поверхностном слое сплава, состав которого для твердого А,В-раствора описывается уравнением равновесной твердофазной адсорбции:

$$
\frac{x_{\mathrm{B}}^{\mathrm{s}}}{x_{\mathrm{A}}^{\mathrm{s}}}=\frac{x_{\mathrm{B}}^{\mathrm{v}}}{x_{\mathrm{A}}^{\mathrm{v}}} \cdot \frac{f_{\mathrm{B}}^{\mathrm{v}} f_{\mathrm{A}}^{\mathrm{s}}}{f_{\mathrm{B}}^{\mathrm{s}} f_{\mathrm{A}}^{\mathrm{v}}} \cdot \mathrm{e}^{-\frac{\Delta \mathrm{G}_{\mathrm{ads}}^{\mathrm{o}}}{\mathrm{RT}}} .
$$

В формуле (2) $f_{\mathrm{i}}^{\mathrm{v}}$ и $f_{\mathrm{i}}^{\mathrm{s}}-$ коэффициенты активности і-го компонента в объеме сплава и на его поверхности соответственно; $\mathrm{R}$ - универсальная газовая постоянная; $\mathrm{T}$ - температура; $\Delta \mathrm{G}_{\mathrm{ads}}^{\circ}$ - стандартная энергия Гиббса твердофазной адсорбции. При условии $\Delta \mathrm{G}_{\mathrm{ads}}^{\circ}<0$ металл В накапливается в поверхностном слое сплава. Такая сегрегация электроположительного компонента характерна для катодно-поляризуемых сплавов системы Ag-Au [3] с преобладанием Ag, находящихся в контакте с водными растворами поверхностно-инактивных электролитов, предварительно подвергнутыми глубокой деаэрации. При этом исключается избирательное растворение серебра. Предполагается [3], что к относительному увеличению термодинамической активности $\mathrm{Au}$ в поверхностном слое $\mathrm{Ag}$, Au-сплава приводит более высокая гидрофильность золота. В пользу такого предположения свидетельствуют данные о повышении емкости плотной части двойного электрического слоя при переходе от серебра к золоту. С другой стороны, по данным [4,5] в Ag,Au-сплавах, в которых основным компонентом является уже золото, поверхностно-активным компонентом сплава является $\mathrm{Ag}$, которое, по мнению авторов, «выжимается» на границу с раствором за счет более низкой удельной работы образования поверхности.

Как правило, процесс СР твердых растворов замещения начинает лимитироваться твердофазной диффузией уже через несколько секунд после начала анодной поляризации. Большинство развитых к настоящему моменту физико-химических моделей массопереноса в твердой фазе сплава учитывают определяющую роль неравновесной вакансионной подсистемы, формирующейся при ионизации электроотрицательного компонента. В то же время равновесный эффект твердофазной адсорбции компонентов в поверхностном слое сплава [3-6] в кинетике СР не учитывался. 
Цель работы: решение нестационарной задачи диффузии при потенциостатическом селективном растворении гомогенного сплава, сопровождающемся предварительным твердофазным адсорбционным накоплением одного из компонентов в поверхностном слое.

\section{Теоретическая часть}

Запишем исходную систему уравнений нестационарной полубесконечной диффузии при СР бинарного сплава. Учтем, что на неподвижной плоской межфазной поверхности сплав/электролит происходит скачкообразное адсорбционное изменение атомной доли электроотрицательного компонента А. Независимо от режима электролиза решение дифференциального уравнения

$$
\frac{\partial x_{\mathrm{A}}(\mathrm{z}, \mathrm{t})}{\partial \mathrm{t}}=\mathrm{D}_{\mathrm{A}} \frac{\partial^{2} x_{\mathrm{A}}(\mathrm{z}, \mathrm{t})}{\partial \mathrm{z}^{2}}
$$

позволяет найти пространственно-временной профиль концентрации диффузанта $x_{\mathrm{A}}(\mathrm{z}, \mathrm{t})$. В уравнении (3) $\mathrm{z}$ - координата, нормальная поверхности электрода; $\mathrm{t}-$ время; $\mathrm{D}_{\mathrm{A}}-$ коэффициент диффузии. Начальное условие задачи

$$
\left.x_{\mathrm{A}}(\mathrm{z}, \mathrm{t})\right|_{\mathrm{t}=0}=x_{\mathrm{A}}^{\mathrm{v}}\left\{1+\left[\Phi\left(x_{\mathrm{A}}^{\mathrm{v}} ; \mathrm{K}_{\mathrm{ads}}\right)-1\right] \cdot \delta(\mathrm{z})\right\}
$$

включает наряду с объемной атомной долей электроотрицательного компонента $x_{\mathrm{A}}^{\mathrm{v}}$ импульсную функцию $\delta(\mathrm{z})$, которая равна единице при $\mathrm{z}=0$. При этом согласно условию (4) поверхностная концентрация компонента А принимает значение, равное $x_{\mathrm{A}}^{\mathrm{v}} \cdot \Phi\left(x_{\mathrm{A}}^{\mathrm{v}} ; \mathrm{K}_{\mathrm{ads}}\right)$. Равновесная изотерма адсорбции $\Phi\left(x_{\mathrm{A}}^{\mathrm{v}} ; \mathrm{K}_{\mathrm{ads}}\right) \neq 1$ включает константу твердофазного адсорбционного равновесия $K_{\mathrm{ads}}$ и определяет степень обогащения поверхностного слоя сплава одним из компонентов еще до начала процесса СР. Изменением приповерхностной концентрации ионов $\mathrm{A}^{\mathrm{n}_{\mathrm{A}}}$ за счет адсорбции в растворе пренебрегаем.

Первое граничное условие задает значение концентрации на достаточно большом удалении от поверхности раздела сплав/электролит

$$
\left.x_{\mathrm{A}}(\mathrm{z}, \mathrm{t})\right|_{\mathrm{z} \rightarrow \infty}=x_{\mathrm{A}}^{\mathrm{v}} \text {, }
$$

в то время как форма второго граничного условия зависит от режима электролиза, ибо могут быть заданы поток вещества или его концентрация на межфазной границе. В потенциостатическом режиме поляризации выполняется условие постоянства поверхностной концентрации диффузанта:

$$
\left.x_{\mathrm{A}}(\mathrm{z}, \mathrm{t})\right|_{\mathrm{z}=0}=x_{\mathrm{A}}^{\mathrm{v}} \cdot \Phi\left(x_{\mathrm{A}}^{\mathrm{v}} ; \mathrm{K}_{\text {ads }}\right) \cdot \mathrm{e}^{-\frac{\mathrm{n}_{\mathrm{A}} \mathrm{F}}{\mathrm{RT}} \eta}=\text { const },
$$

в котором $\eta$ - перенапряжение; F - число Фарадея. Соответствующий транзиент парциального тока растворения компонента А при $\eta=$ const полностью определяется величиной его нестационарного диффузионного потока:

$$
\mathrm{I}_{\mathrm{A}}(\mathrm{t})=\left.\frac{\mathrm{n}_{\mathrm{A}} \mathrm{FD}_{\mathrm{A}} \mathrm{S}_{\mathrm{g}}}{\mathrm{V}_{\mathrm{m}}^{\text {all }}} \cdot \frac{\partial x_{\mathrm{A}}(\mathrm{z}, \mathrm{t})}{\partial \mathrm{z}}\right|_{\mathrm{z}=0},
$$

где $\mathrm{S}_{\mathrm{g}}$ - геометрическая (видимая) площадь электрода, a $\mathrm{V}_{\mathrm{m}}^{\text {all }}$ - молярный объем сплава. 
Поиск $x_{\mathrm{A}}(\mathrm{z}, \mathrm{t})$ и $\mathrm{I}_{\mathrm{A}}(\mathrm{t})$ вели, предварительно подвергнув исходную систему уравнений (3)-(7) интегральному преобразованию Лапласа-Карсона [7], вводя комплексные функции изображений концентрационного профиля и силы тока:

$$
\begin{aligned}
\bar{x}_{\mathrm{A}}(\mathrm{z}, \mathrm{p}) & =\mathrm{p} \cdot \int_{0}^{\infty} x_{\mathrm{A}}(\mathrm{z}, \mathrm{t}) \mathrm{e}^{-\mathrm{pt}} \mathrm{dt}, \\
\overline{\mathrm{I}}_{\mathrm{A}}(\mathrm{p}) & =\mathrm{p} \cdot \int_{0}^{\infty} \mathrm{I}_{\mathrm{A}}(\mathrm{t}) \mathrm{e}^{-\mathrm{pt}} \mathrm{dt} .
\end{aligned}
$$

Это позволило уменьшить число независимых аргументов, ибо в функции $\bar{x}_{\mathrm{A}}(\mathrm{z}, \mathrm{p})$ и $\overline{\mathrm{I}}_{\mathrm{A}}(\mathrm{p})$ входит теперь не независимая переменная времени, а параметр преобразования р. Система уравнений (3)-(7), преобразованная по Лапласу-Карсону, включает теперь обыкновенное дифференциальное уравнение второго порядка и принимает следующий вид:

$$
\begin{gathered}
\mathrm{p}\left[\bar{x}_{\mathrm{A}}(\mathrm{z}, \mathrm{p})-x_{\mathrm{A}}^{\mathrm{v}}-\left(x_{\mathrm{A}}^{\mathrm{v}} \Phi\left(x_{\mathrm{A}}^{\mathrm{v}} ; \mathrm{K}_{\mathrm{ads}}\right)-x_{\mathrm{A}}^{\mathrm{v}}\right) \delta(\mathrm{z})\right]=\mathrm{D}_{\mathrm{A}} \frac{\mathrm{d}^{2} \bar{x}_{\mathrm{A}}(\mathrm{z}, \mathrm{p})}{\mathrm{dz}^{2}}, \\
\left.\bar{x}_{\mathrm{A}}(\mathrm{z}, \mathrm{p})\right|_{\mathrm{z} \rightarrow \infty}=x_{\mathrm{A}}^{\mathrm{v}}, \\
\left.\bar{x}_{\mathrm{A}}(\mathrm{z}, \mathrm{p})\right|_{\mathrm{z}=0}=x_{\mathrm{A}}^{\mathrm{v}} \cdot \Phi\left(x_{\mathrm{A}}^{\mathrm{v}} ; \mathrm{K}_{\mathrm{ads}}\right) \cdot \mathrm{e}^{-\frac{\mathrm{n}_{\mathrm{A}} \mathrm{F}}{\mathrm{RT}} \eta}, \\
\overline{\mathrm{I}}_{\mathrm{A}}(\mathrm{p})=\left.\frac{\mathrm{n}_{\mathrm{A}} \mathrm{FD}_{\mathrm{A}} \mathrm{S}_{\mathrm{g}}}{\mathrm{V}_{\mathrm{m}}^{\mathrm{all}}} \cdot \frac{\mathrm{d} \bar{x}_{\mathrm{A}}(\mathrm{z}, \mathrm{p})}{\mathrm{dz}}\right|_{\mathrm{z}=0} .
\end{gathered}
$$

Решением данной системы уравнений являются выражения для концентрационного профиля и силы тока в пространстве изображений:

$$
\begin{gathered}
\bar{x}_{\mathrm{A}}(\mathrm{z}, \mathrm{p})=x_{\mathrm{A}}^{\mathrm{v}}\left[1+\left(\Phi\left(x_{\mathrm{A}}^{\mathrm{v}} ; \mathrm{K}_{\text {ads }}\right)-1\right) \cdot \delta(\mathrm{z})\right]+x_{\mathrm{A}}^{\mathrm{v}} \cdot \Phi\left(x_{\mathrm{A}}^{\mathrm{v}} ; \mathrm{K}_{\mathrm{ads}}\right) \cdot\left[\mathrm{e}^{\left.-\frac{\mathrm{n}_{\mathrm{A}} \mathrm{F}}{\mathrm{RT}}-1\right] \cdot \mathrm{e}^{-\mathrm{z} \frac{\mathrm{p}^{1 / 2}}{\left(\mathrm{D}_{\mathrm{A}}\right)^{1 / 2}}},}\right. \\
\overline{\mathrm{I}}_{\mathrm{A}}(\mathrm{p})=\frac{\mathrm{n}_{\mathrm{A}} \mathrm{F} x_{\mathrm{A}}^{\mathrm{v}}\left(\mathrm{D}_{\mathrm{A}}\right)^{1 / 2} \mathrm{p}^{1 / 2} \mathrm{~S}_{\mathrm{g}}}{\mathrm{V}_{\mathrm{m}}^{\text {all }}}\left[1-\mathrm{e}^{-\frac{\mathrm{n}_{\mathrm{A}} \mathrm{F}}{\mathrm{RT}} \eta}\right] \cdot \Phi\left(x_{\mathrm{A}}^{\mathrm{v}} ; \mathrm{K}_{\text {ads }}\right) .
\end{gathered}
$$

Обратное преобразование этих выражений приводит к соотношениям, описывающим соответственно концентрационное поле металла А в сплаве

$$
x_{\mathrm{A}}(\mathrm{z}, \mathrm{t})=x_{\mathrm{A}}^{\mathrm{v}} \cdot\left[1+\left(\Phi\left(x_{\mathrm{A}}^{\mathrm{v}} ; \mathrm{K}_{\mathrm{ads}}\right)-1\right) \delta(\mathrm{z})-\Phi\left(x_{\mathrm{A}}^{\mathrm{v}} ; \mathrm{K}_{\text {ads }}\right)\left[1-\mathrm{e}^{-\frac{\mathrm{n}_{\mathrm{A}} \mathrm{F}}{\mathrm{RT}} \eta}\right] \mathrm{e}^{-\frac{\mathrm{z}}{\left(\pi \mathrm{D}_{\mathrm{A}} \mathrm{t}\right)^{1 / 2}}}\right]
$$

и токовый транзиент

$$
\mathrm{I}_{\mathrm{A}}(\mathrm{t})=\frac{\mathrm{n}_{\mathrm{A}} \mathrm{F} x_{\mathrm{A}}^{\mathrm{v}}\left(\mathrm{D}_{\mathrm{A}}\right)^{1 / 2} \mathrm{~S}_{\mathrm{g}}}{\mathrm{V}_{\mathrm{m}}^{\text {all }} \pi^{1 / 2} \mathrm{t}^{1 / 2}}\left[1-\mathrm{e}^{-\frac{\mathrm{n}_{\mathrm{A}} \mathrm{F}}{\mathrm{RT}} \eta}\right] \cdot \Phi\left(x_{\mathrm{A}}^{\mathrm{v}} ; \mathrm{K}_{\mathrm{ads}}\right) .
$$

Как и следовало ожидать, фигурирующая в них функция изотермы равновесной адсорбции $\Phi\left(x_{\mathrm{A}}^{\mathrm{v}} ; \mathrm{K}_{\mathrm{ads}}\right)$ полностью ответственна за вклад эффекта предполяризационного сегрегационного накопления компонентов в значение силы парциального тока процесса $\mathrm{CP}$, контролируемого твердофазной диффузией металла А в сплаве.

\section{Эксперимент}

Для исследования выбраны поликристаллические гомогенные $\mathrm{Ag}, \mathrm{Au}-$-плавы $\left(x_{\mathrm{Au}}^{\mathrm{v}}=5 \div 35\right.$ ат.\%) со статистически неупорядоченной микроструктурой, из которых 
изготовлены массивные плоские электроды. Растворение таких сплавов в кислых нитратных средах при наличии ионов $\mathrm{Ag}^{+}$в достаточном количестве характеризуется высоким током обмена стадии $\mathrm{Ag}^{+}+\mathrm{e}^{-} \leftrightarrow \mathrm{Ag}[8]$, при не слишком высоких анодных потенциалах протекает в активном состоянии и не осложнено комплексообразованием. Поскольку Au в этих условиях остается термодинамически устойчивым, а фарадеевские токи побочных процессов в хорошо деаэрированных растворах ничтожно малы, то растворение можно считать истинно селективным.

Сплавы системы Ag-Au относятся к тем немногим, для которых установлена равновесная изотерма твердофазной адсорбции компонентов на границе с водным раствором поверхностно-инактивных анионов $\mathrm{F}^{-}$и $\mathrm{ClO}_{4}^{-}$[3]; с определенным допущением к таковым можно отнести и $\mathrm{NO}_{3}{ }^{-}$.

Твердые растворы серебра (99.99\%) с золотом (99.99\%) получены прямым сплавлением в вакуумированных кварцевых ампулах, последующим отжигом в течение двух часов при 1213 К и закалкой в воду. При изготовлении электродов сплавы механически разрезали, шлифовали и помещали в оправу из эпоксидной смолы.

Подготовка поверхности электродов к опыту включала зачистку на корундовой шлифовальной бумаге, полировку на замше с водной суспензией оксида магния, обезжиривание этиловым спиртом и промывание бидистиллятом.

Нитратные растворы $0.1 \mathrm{M} \mathrm{NaNO}_{3}+0.001 \mathrm{M} \mathrm{HNO}_{3}+\mathrm{x} \mathrm{M} \mathrm{AgNO} 3\left(\mathrm{x}=10^{-4}, 10^{-3}\right.$, $10^{-2}$ ) готовили из ч. д. а. реактивов на бидистилляте и деаэрировали аргоном. Потенциостатические исследования выполняли при 298 К в стеклянной электрохимической ячейке с неразделенными пространствами рабочего и вспомогательного $(\mathrm{Pt})$ электродов, регистрируя хроноамперограммы при неизменном перенапряжении $\eta$ в течение 20 мин. Хлоридсеребряный электрод сравнения находился в отдельном сосуде и соединялся с ячейкой электролитическим ключом, заполненным $\mathrm{NH}_{4} \mathrm{NO}_{3}$.

\section{Обсуждение результатов}

Из сопоставления соотношения (14) и уравнения Коттреля [9]

$$
I_{A}(t)=\frac{n_{A} F x_{A}^{v}\left(D_{A}\right)^{1 / 2} S_{g}}{V_{m}^{\text {all }} \pi^{1 / 2} t^{1 / 2}}\left[1-e^{-\frac{n_{A} F}{R T} \eta}\right],
$$

описывающего токовый транзиент диффузионно-контролируемого СР бинарного сплава в отсутствие твердофазного адсорбционного эффекта, видно, что без учета изменения концентраций компонентов в поверхностном слое значения коэффициента диффузии $\mathrm{D}_{\mathrm{A}}$, определенные по кривой спада тока с использованием (2), будут заниженными (когда $\Phi\left(x_{\mathrm{A}}^{\mathrm{v}} ; \mathrm{K}_{\text {ads }}\right)<1$ ) или завышенными (когда $\Phi\left(x_{\mathrm{A}}^{\mathrm{v}} ; \mathrm{K}_{\mathrm{ads}}\right)>1$ ) в $\left[\Phi\left(x_{\mathrm{A}}^{\mathrm{v}} ; \mathrm{K}_{\mathrm{ads}}\right)\right]^{2}$ раз.

Для ответа на вопрос, насколько коэффициент диффузии серебра чувствителен к изменению поверхностного состава $\mathrm{Ag}$, $\mathrm{Au}$-сплава, которое проявляется еще до начала $\mathrm{CP}$, рассчитали коэффициенты диффузии $\mathrm{Ag}$ в сплаве $\mathrm{D}_{\mathrm{Ag}}$ по формулам (14) и (15), используя прямолинейные участки опытных хроноамперограмм, перестроенных в координатах $\mathrm{I}_{\mathrm{Ag}} / \mathrm{S}_{\mathrm{g}}-\mathrm{t}^{-1 / 2}$. Учитывали, что функция $\Phi\left(x_{\mathrm{Ag}}^{\mathrm{v}} ; \mathrm{K}_{\mathrm{ads}}\right)$ для рассматриваемой металлической системы принимает следующий вид [3]:

$$
\Phi\left(x_{\mathrm{Ag}}^{\mathrm{v}} ; \mathrm{K}_{\mathrm{ads}}\right)=\left\{x_{\mathrm{Ag}}^{\mathrm{v}}+\mathrm{K}_{\mathrm{ads}}\left(1-x_{\mathrm{Ag}}^{\mathrm{v}}\right) \exp \left[-2 \chi \frac{x_{\mathrm{Ag}}^{\mathrm{v}}\left(1-x_{\mathrm{Ag}}^{\mathrm{v}}\right)\left(\mathrm{K}_{\mathrm{ads}}-1\right)}{1+\mathrm{K}_{\mathrm{ads}}\left(1-x_{\mathrm{Ag}}^{\mathrm{v}}\right)}\right]\right\}^{-1} .
$$


Здесь параметр $\chi$ учитывает характер межатомных взаимодействий в сплаве. Для системы $\mathrm{Ag}-\mathrm{Au} \mid \mathrm{ClO}_{4}^{-}\left(\mathrm{H}_{2} \mathrm{O}\right)$ в [3] найдено, что $\chi=1.21 \pm 0.13 ; \mathrm{K}_{\mathrm{ads}}=1.26 \pm 0.14$; в случае $\mathrm{Ag}-\mathrm{Au} \mid \mathrm{F}^{-}\left(\mathrm{H}_{2} \mathrm{O}\right)$ параметры $\chi=1.60 \pm 0.10 ; \mathrm{K}_{\mathrm{ads}}=1.46 \pm 0.12$. Эти данные указывают, что твердый раствор золота в серебре не идеален и характеризуется небольшими отрицательными отклонениями от закона Рауля $(\chi>0)$, а повышенную поверхностную активность проявляет золото $\left(\mathrm{K}_{\mathrm{ads}}>1\right)$; как следствие, функция $\Phi\left(x_{\mathrm{Ag}}^{\mathrm{v}} ; \mathrm{K}_{\mathrm{ads}}\right)<1$. В пределах погрешности природа поверхностно инактивного аниона $\left(\mathrm{ClO}_{4}^{-}\right.$или $\left.\mathrm{F}^{-}\right)$почти не влияет на значение $\mathrm{K}_{\mathrm{ads}}$ и $\chi$. Поэтому при поиске $\Phi\left(x_{\mathrm{Ag}}^{\mathrm{v}} ; \mathrm{K}_{\mathrm{ads}}\right)$ для системы $\mathrm{Ag}-\mathrm{Au} \mid \mathrm{NO}_{3}^{-}\left(\mathrm{H}_{2} \mathrm{O}\right)$ использовали средние значения этих параметров.

Расчет показывает, что учет равновесной твердофазной адсорбции компонентов в поверхностном слое $\mathrm{Ag}, \mathrm{Au}-$ сплавов в рамках представленной модели не приводит к существенным изменениям в величине $\mathrm{D}_{\mathrm{Ag}}$ (таблица). Однако из-за того, что $\Phi\left(x_{\mathrm{Ag}}^{\mathrm{v}} ; \mathrm{K}_{\mathrm{ads}}\right)<1$, значения $\mathrm{D}_{\mathrm{Ag}}$, скорректированные на эффект сегрегации, оказываются все же несколько выше найденных по наиболее простой модели Коттреля. Различие уменьшается с ростом концентрации серебра в сплаве (таблица), так как при этом снижается и степень относительного обогащения поверхности сплава золотом [3]. Очевидно, данный эффект следует учитывать при прецизионном определении коэффициентов твердофазной диффузии в поверхностном слое гомогенного сплава, формирующемся при его селективном растворении.

Таблица. Коэффициенты диффузии серебра в сплавах системы Ag-Au, рассчитанные по хроноамперометрическим данным без учета (числитель) и с учетом (знаменатель) эффекта твердофазной адсорбции золота

\begin{tabular}{|c|c|c|c|}
\hline$x_{\mathrm{Au}}^{\mathrm{v}}$ & $\Phi\left(x_{\mathrm{Ag}}^{\mathrm{v}} ; \mathrm{K}_{\mathrm{ads}}\right)$ & $\eta, B$ & $\mathrm{D}_{\mathrm{Ag}} \cdot 10^{18}, \mathrm{~cm}^{2} / \mathrm{c}$ \\
\hline 0.05 & 0.985 & 0.1 & $185 / 191$ \\
\hline 0.10 & 0.976 & 0.1 & $75.1 / 78.9$ \\
\hline \multirow{3}{*}{0.20} & \multirow{3}{*}{0.968} & 0.1 & $4.40 / 4.70$ \\
\hline & & 0.2 & $20.7 / 22.1$ \\
\hline & & 0.3 & $83.8 / 89.5$ \\
\hline \multirow{3}{*}{0.30} & \multirow{3}{*}{0.964} & 0.1 & $1.12 / 1.20$ \\
\hline & & 0.2 & $4.32 / 4.65$ \\
\hline & & 0.3 & $14.8 / 15.9$ \\
\hline \multirow{3}{*}{0.35} & \multirow{3}{*}{0.963} & 0.1 & $0.82 / 0.88$ \\
\hline & & 0.2 & $4.38 / 4.72$ \\
\hline & & 0.3 & $12.5 / 13.4$ \\
\hline
\end{tabular}

\section{Заключение}

Математическое моделирование эффекта твердофазной адсорбции при анодном селективном растворении гомогенного сплава в режиме диффузионной кинетики показывает, что сегрегационное изменение поверхностной концентрации сплава приводит к пропорциональному изменению наклона критериальной коттрелевой зависимости скорости процесса, выраженной в токовых единицах, от времени. Учет равновесной адсорбции компонентов в поверхностном слое $\mathrm{Ag}$, $\mathrm{Au}-$ сплавов с $x_{\mathrm{Au}}^{\mathrm{v}} \leq 0.35$ до начала СР необходим при прецизионном определении диффузионных 
атомарных подвижностей, ибо соответствующая корректировка приводит к увеличению расчетных величин коэффициента диффузии. Поправка тем более заметна, чем выше содержание золота в объеме сплава.

Работа выполнена при поддержке Минобрнауки России в рамках государственного задания ВУЗам в сфере научной деятельности на 2014-2016 г2. (проект № 951).

\section{Список литературы}

1. Landolt D. Corrosion and surface chemistry of metals. Lausanne (Switzerland), EPFL Press, 2007. $632 \mathrm{p}$.

2. Бокштейн Б.С., Копецкий Ч.В., Швиндлерман Л.С. Термодинамика и кинетика грании зерен в металлах. М. Металлургия. 1986. $224 \mathrm{c}$.

3. Бобринская Е.В., Введенский А.В. // Электрохимия. 2001. Т. 37. № 9. С. 11021106.

4. Сафонов В.А., Чоба М.А., Серопегин Ю.Д., Лубнин Е.Н. // Электрохимия. 2006. Т. 42, № 8. С. 957-968.

\section{References}

1. Landolt D. Corrosion and surface chemistry of metals. Lausanne (Switzerland), EPFL Press, 2007, 632 p.

2. Bokshtein B.S., Kopetskii Ch.V., Shvindlerman L.S. Termodinamika $i$ kinetika granits zeren $v$ metallakh. Moskva, Metallurgiya, 1986, $224 \mathrm{p}$.

3. Bobrinskaya E.V., Vvedenskii A.V., Elektrokhimiya, 2001, Vol. 37, No 9, pp. 1102-1106.

4. Safonov V.A., Choba M.A., Seropegin Yu.D., Lubnin E.N., Elektrokhimiya, 2006, Vol. 42, No 8, pp. 957-968.

5. Manzhos R.A., Krivenko A.G., Doronin S.V., Choba M.A. et al., J. Electroanal. Chem.,

Козадеров Олег Александрович - к.х.н., доцент, доцент кафедры физической химии химического факультета, Воронежский государственный университет; Воронеж, тел. (473)2208538

Введенский Александр Викторович - д.х.н., профессор, заведующий кафедрой физической химии химического факультета, Воронежский государственный университет; Воронеж

Селеменев Владимир Федорович - д.х.н., професcop, заведующий кафедрой аналитической химии химического факультета, Воронежский государственный университет; Воронеж
5. Manzhos R.A., Krivenko A.G., Doronin S.V., Choba M.A. et al. // J. Electroanal. Chem. 2013. Vol. 704. pp. 175-182.

6. Кукк Ю.А., Клавилье Ж. // Электрохимия. 1977. Т. 13. № 6. С. 841-844.

7. Диткин В.А., Прудников А.П. Интегральные преобразования и операционное исчисление. М. Наука, 1974. 544 с.

8. Сухотин А.М. Справочник по электрохимии. Ленинград. Химия. 1981. 486 с.

9. Bard A.J., Faulkner L.R. Electrochemical methods. Fundamentals and applications. NewYork (USA). Wiley. 2000. 856 p.

2013, Vol. 704, pp. 175-182. DOI: 10.1016/j.jelechem.2013.07.010

6. Kukk Yu., Clavilier J., Elektrokhimiya, 1977, Vol. 13, No 6, pp. 841-844.

7. Ditkin V.A., Prudnikov A.P., Integral'nye preobrazovaniya $i$ operatsionnoe ischislenie., M., Nauka, 1974, 544 p.

8. Sukhotin A.M. Spravochnik po elektrokhimii. Leningrad, Khimiya, 1981, 486 p.

9. Bard A.J., Faulkner L.R., Electrochemical methods. Fundamentals and applications. NewYork (USA), Wiley, 2000, 856 p.

Kozaderov Oleg A. - PhD (Chem.), Associate Professor, Chemical Faculty, Physical Chemistry Department, Voronezh State University; Voronezh, e-mail: ok@chem.vsu.ru

Vvedenskii Aleksandr V. - Dr. Sci. (Chemistry), Professor, head of Department of Physical Chemistry, Chemical Faculty, Voronezh State University, Voronezh; Voronezh, e-mail: alvved@chem.vsu.ru

Selemenev Vladimir F. - Dr. Sci. (Chemistry), Professor, head of Department of Analytical Chemistry, Chemical Faculty, Voronezh State University, Voronezh; Voronezh, e-mail: common@chem.vsu.ru 